\title{
Nobel Lecture: The invention and early history of the CCD*
}

\author{
George E. Smith \\ Bell Laboratories, Murray Hill, New Jersey 08005-2822, USA
}

(Published 13 August 2010)

DOI: $10.1103 /$ RevModPhys.82.2307

To try to indicate how the invention of charge coupled devices (CCDs) in 1969 by W. S. Boyle and myself came about, it is first necessary to describe three important technologies being pursued at the time. Magnetic bubbles, the silicon diode array vidicon for the Picturephone, and metal oxide semiconductor (MOS) technology were all contributing factors to the invention of the device.

Magnetic bubbles were formed by placing a special magnetic material, typically garnet, in a magnetic field so that circular magnetic domains could be formed as shown in Fig. 1. A Permalloy pattern was deposited on the surface which could capture the domain, and when a rotating magnetic field was applied in the plane of the slice, the bubble would be shifted along from one site to the next. With an input device to inject the presence or absence of bubbles representing 0's or 1's and a detection device at the end, one had a serial memory device.

The second driving technology being worked on, which just happened to be in my department, was the silicon diode array camera tube, shown in Fig. 2. This was being developed for the Picturephone, which AT\&T-Bell Labs parent and the major telephone provider at the time - thought would be a huge market once people discovered they could see as well as hear one another over the existing twisted pair phone lines. The initial imaging device considered was the standard commercial vidicon with an antimony trisulfide coating on glass accessed by an electron beam as the photosensitive element. This device was notorious for its lack of robustness and longevity. Instead, we were working on a tube with a silicon slice with millions of diodes placed on the surface, which were accessed by an electron beam in a similar manner. The silicon was an improvement; however, we strongly hoped to find an all solid state device to eliminate the electron beam.

The third field being vigorously pursued was MOS transistor technology. This was the technology that finally became the basis of all modern integrated circuits. The metal oxide semiconductor capacitor that forms the gate of the MOS transistor is shown in Fig. 3. Basic studies of this structure were also being carried out in my department.

To understand the atmosphere at the time, one must look at the structure of the Electronics Division at Bell

\footnotetext{
*The 2009 Nobel Prize for Physics was shared by Charles K. Kao, Willard S. Boyle, and George E. Smith. This paper is the text of the address given in conjunction with the award.
}

Labs, which was under Vice President Jack Morton. This was split into two divisions, one for semiconductor devices and one for all others. Bill Boyle was Executive Director of the semiconductor part and I was a Department Head under him. Jack Morton was anxious to speed up the development of magnetic bubbles as a major memory technology, and there was talk of transferring resources from Bill's division to the other where the bubble work was being done. For this not to happen, Morton demanded that Bill's division come up with a semiconductor device to compete with bubbles. To address this demand, on October 17, 1969, Bill and I got together in his office. In a discussion lasting not much more than an hour, the basic structure of the CCD was sketched out on the blackboard, the principles of operation defined, and some preliminary ideas concerning applications were developed. The train of thought evolved as follows.

First, the semiconductor analogy of the magnetic bubble is needed. The electric dual is a packet of charge. The next problem is how to store this charge in a confined region. The structure which came to mind, of course, was the simple MOS capacitor shown in Fig. 3. Charge can be introduced into this depletion region with the amount of charge stored being the magnitude of the signal. To understand this better, a plot of electron energy vs distance into the structure is shown in Fig. 4. As charge is introduced into the depletion region, the potential at the surface rises until the maximum allowable charge is reached. Any further charge added will flow into the substrate.

The last problem was to shift the charge from one site to another, thereby allowing manipulation of the information. This is solved by simply placing the MOS capacitors very close together as shown in Fig. 5, one with

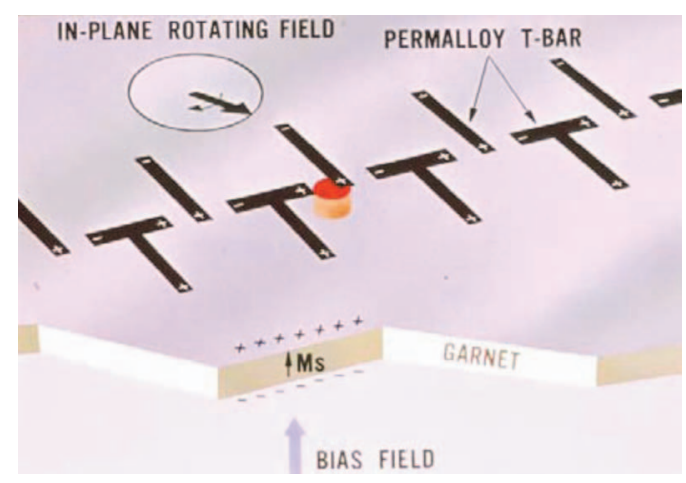

FIG. 1. (Color) Magnetic bubble serial shift register. 


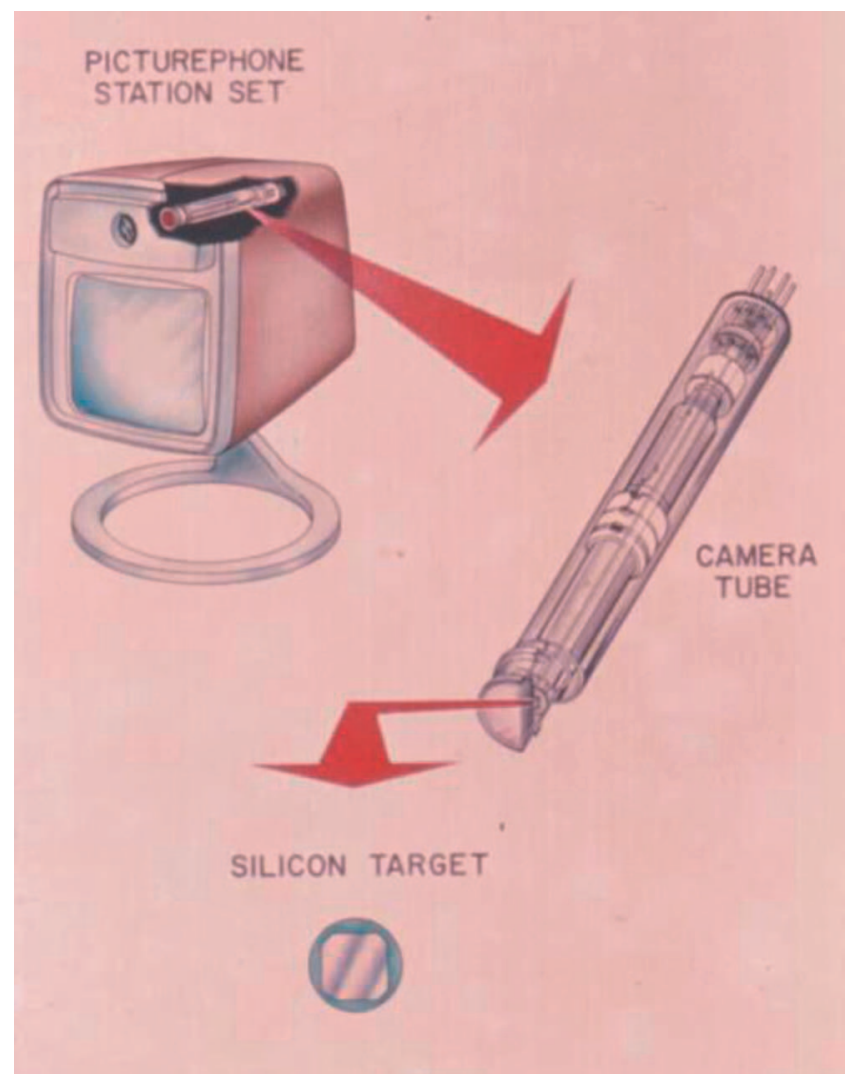

FIG. 2. (Color) The Picturephone set and silicon diode array camera tube.

charge and the second empty. In order to pass the charge from one to the next, one simply applies a more attractive voltage to the second, causing its depletion region to overlap the first and the charge to flow along the surface to the silicon-silicon dioxide interface of the second capacitor.

The original structure using this mechanism to make a shift register is shown in Fig. 6. Many MOS capacitors are placed closely together in a row and connected to a three-phase voltage source. The top figure shows the storage phase with $V_{a}$ applied to one set of electrodes and a smaller rest voltage $V_{b}$ applied to the other two. One site has charge and the other has none. The second figure shows the transfer phase where a larger voltage, $V_{p}$, is applied to the adjacent plates to transfer charge from one to the next. The last two show resetting the voltages to the initial state with the charge information

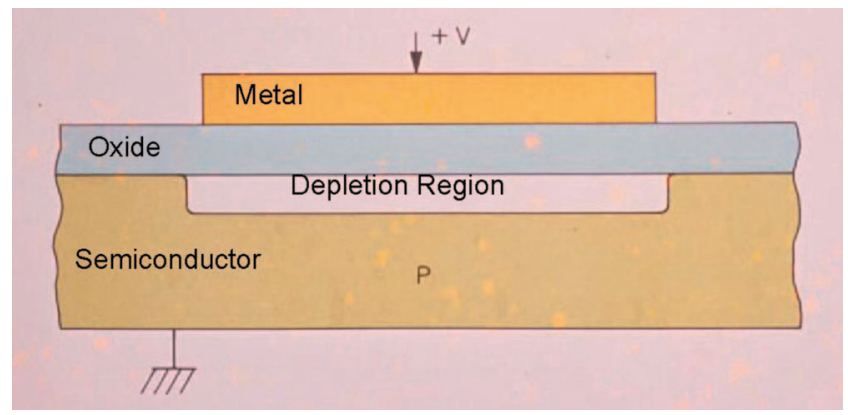

FIG. 3. (Color) The basic MOS structure.

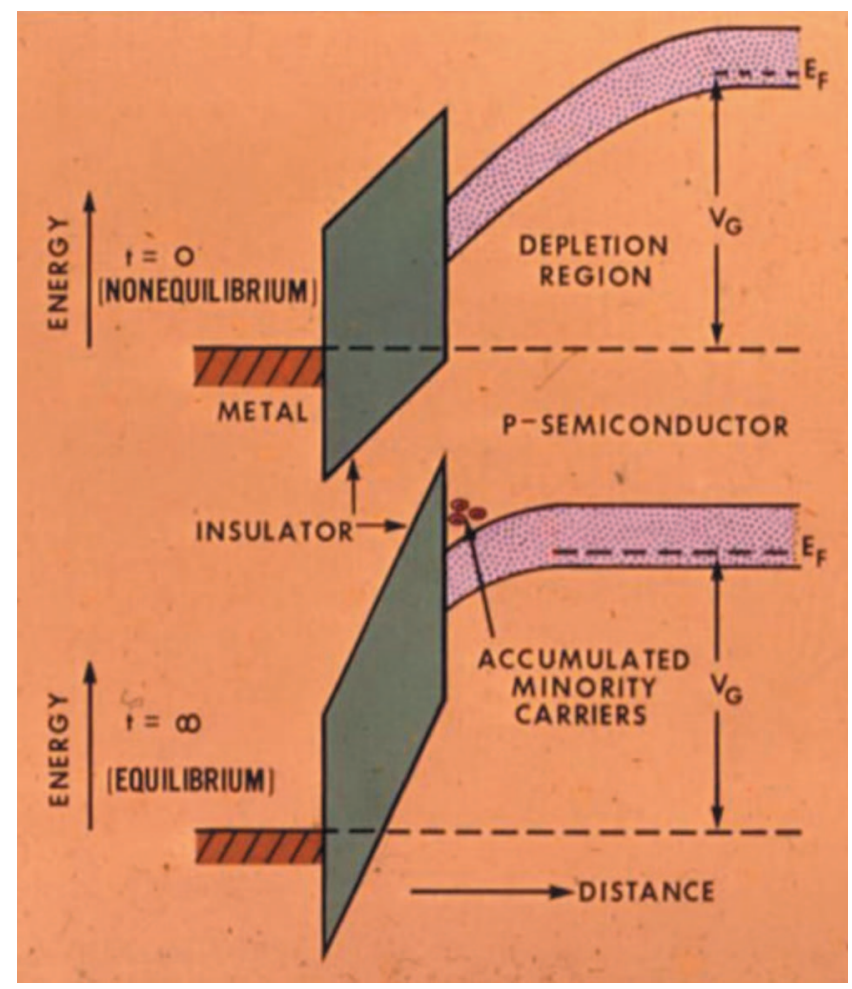

FIG. 4. (Color) A plot of electron energy vs distance through the MOS capacitor.

shifted by one site. This is continued to an output device at the end of the row in order to read the stored information. Many other storage and transfer schemes are possible. The charge can be injected into the device electrically at the beginning of the row making a shift register or supplied by light incident on a structure with empty cells. Then the amount of charge which accumulates by the absorption of photons is determined by the intensity of the light, and the resulting charge pattern can then be read out in shift register fashion after a suitable integration time.

This completed the basic invention. It should be stressed that the basic unit of information in the device was a discrete packet of charge and not the voltages and currents of circuit based devices. The CCD is indeed a functional device and not a collection of individual devices connected by wires.

Finally, it was decided to go ahead and fabricate a device to show experimental feasibility. In less than a

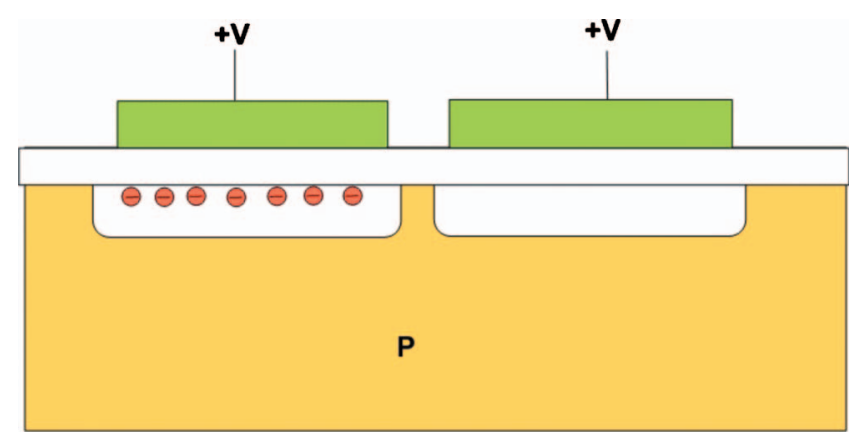

FIG. 5. (Color) Two closely spaced MOS capacitors. 


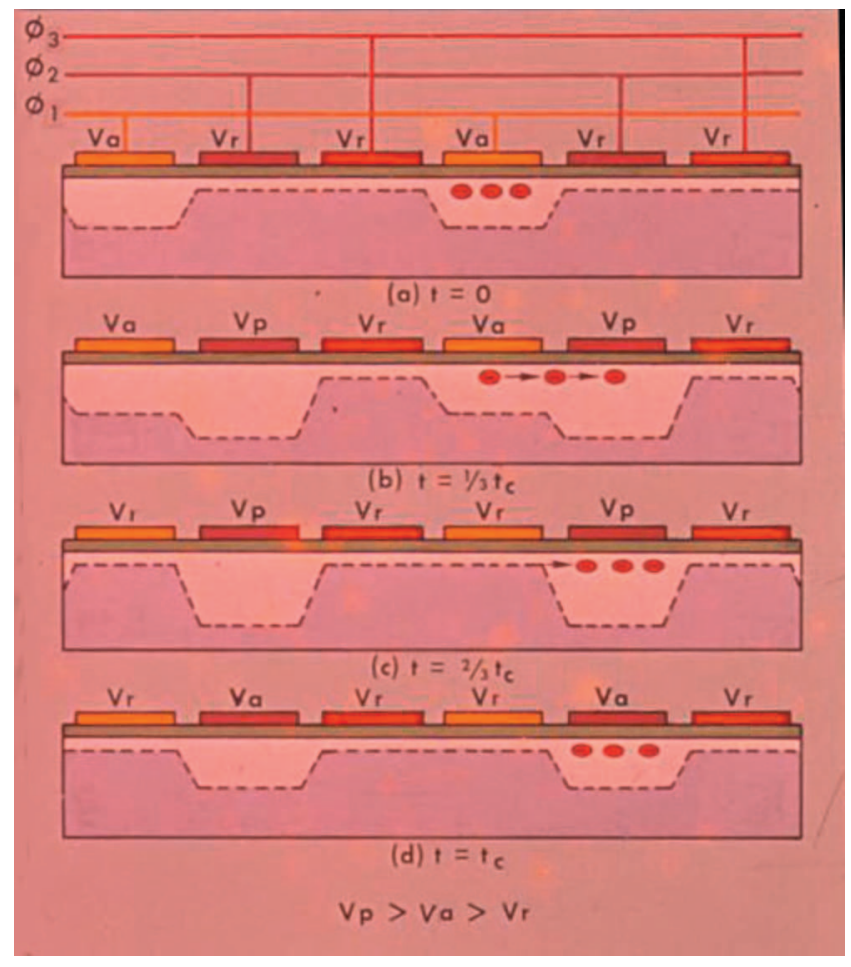

FIG. 6. (Color) The basic CCD structure.

week, masks were made and devices were fabricated and tested. This first simple structure is shown in Fig. 7. Charge was introduced in the first MOS capacitor by thermal generation and then transferred to the output by applying voltages to the plates where it was detected by pushing the charge into the substrate and measuring the substrate current.

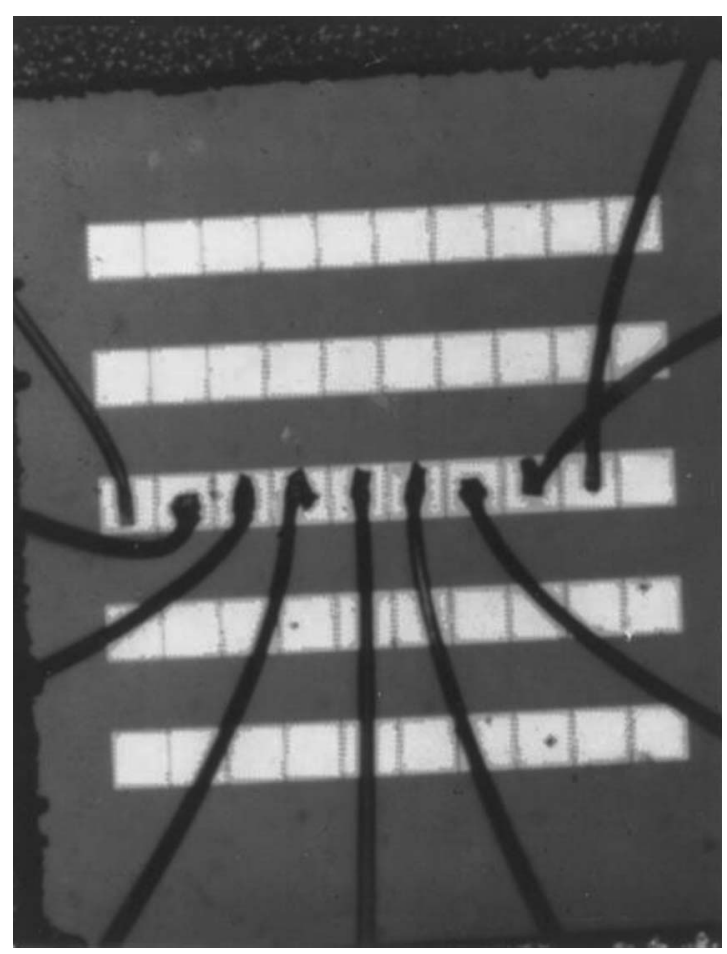

FIG. 7. The first CCD device.

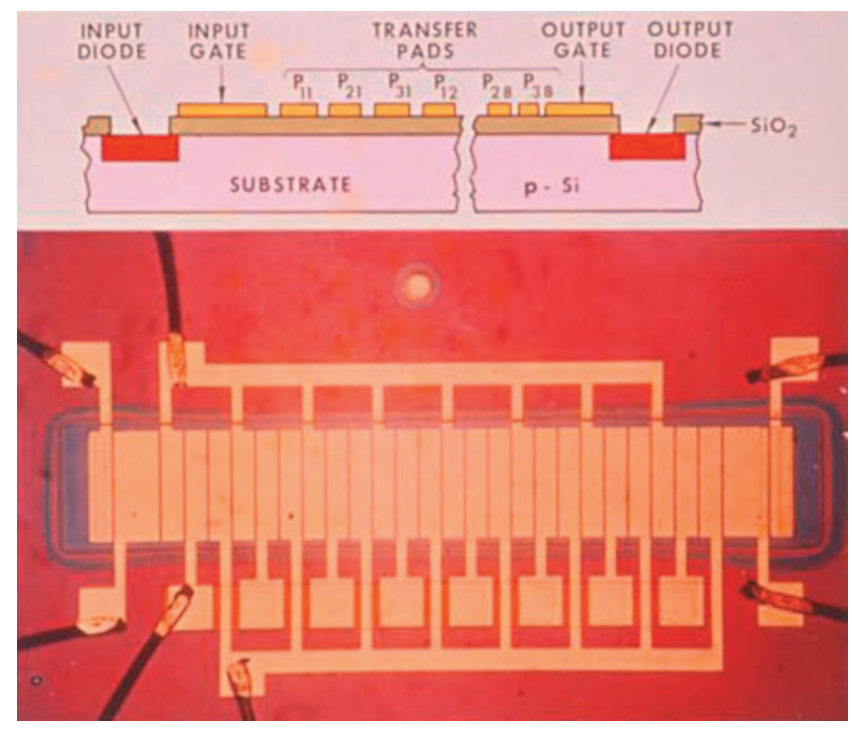

FIG. 8. (Color) The first integrated device.

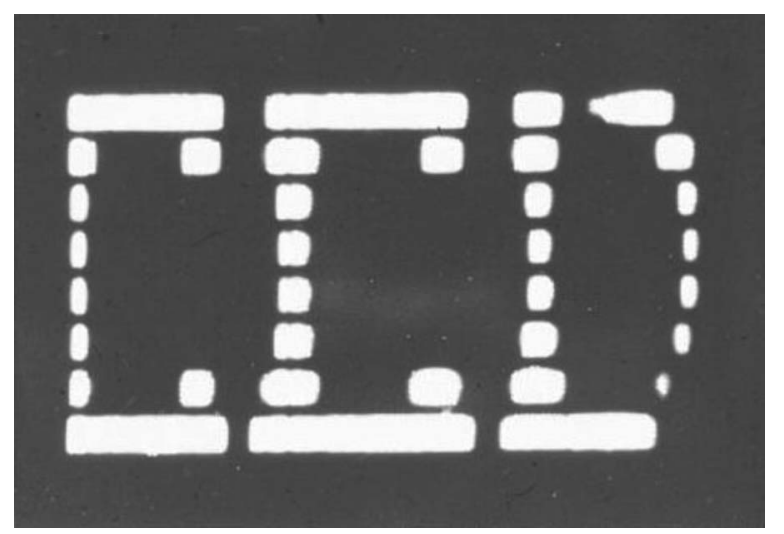

FIG. 9. Oscilloscope display of the output of the 8-bit device used as an imager.

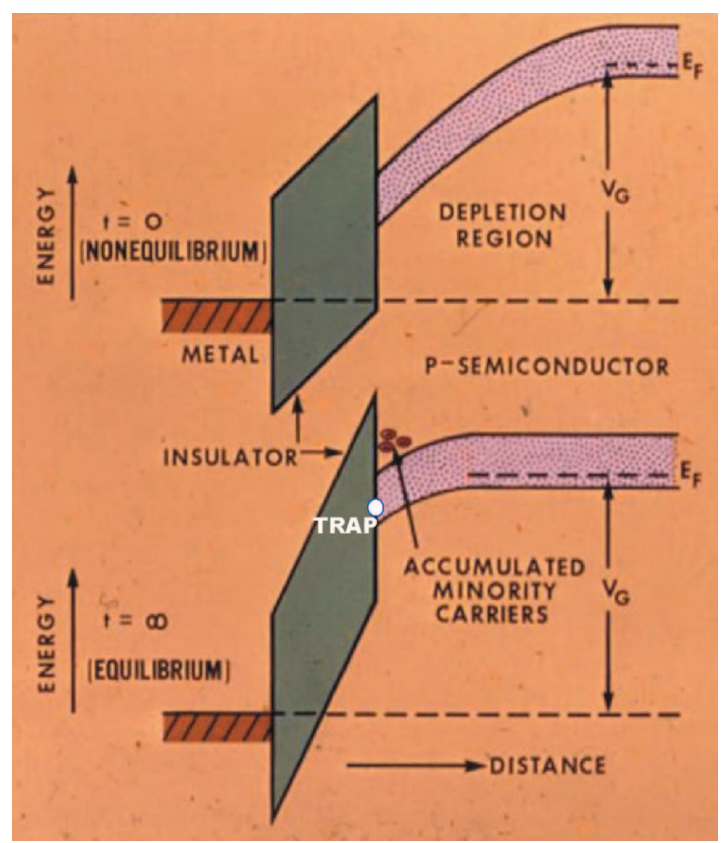

FIG. 10. (Color) Traps at the $\mathrm{Si}-\mathrm{SiO}_{2}$ interface. 

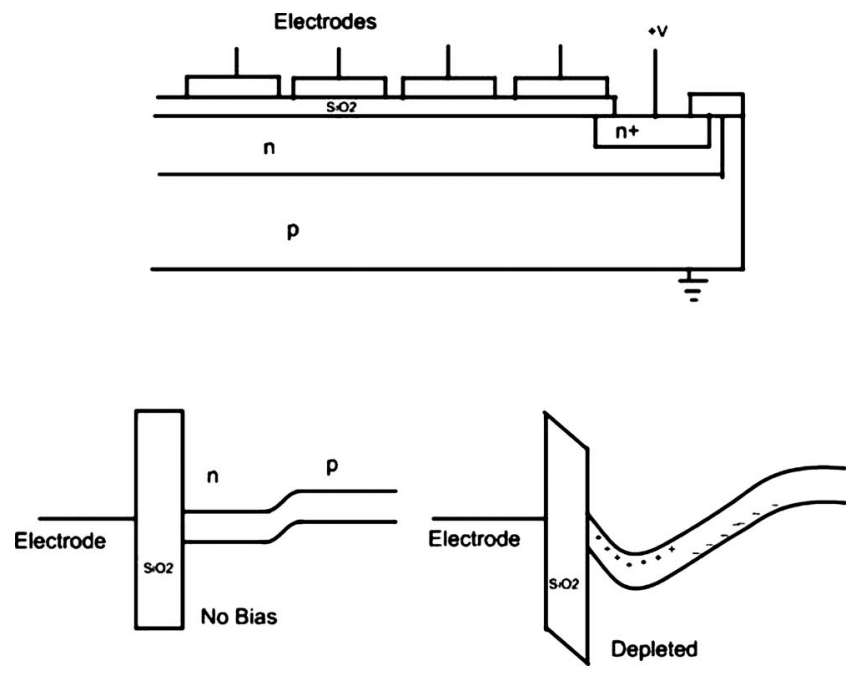

FIG. 11. The buried channel CCD.

The first device was very crude but charge transfer was successfully demonstrated and this was followed by the first integrated structure, which is shown in Fig. 8. This device had a three-phase metallization and diffused input and output successfully demonstrating that it could be operated as a serial memory, the first driving force of the invention. It is no surprise that we tried to use the eight-bit CCD as a linear scanning imaging device and the first rather crude image is shown in Fig. 9.

Following the initial experiments, it was evident that the main problem with the device was charge transfer

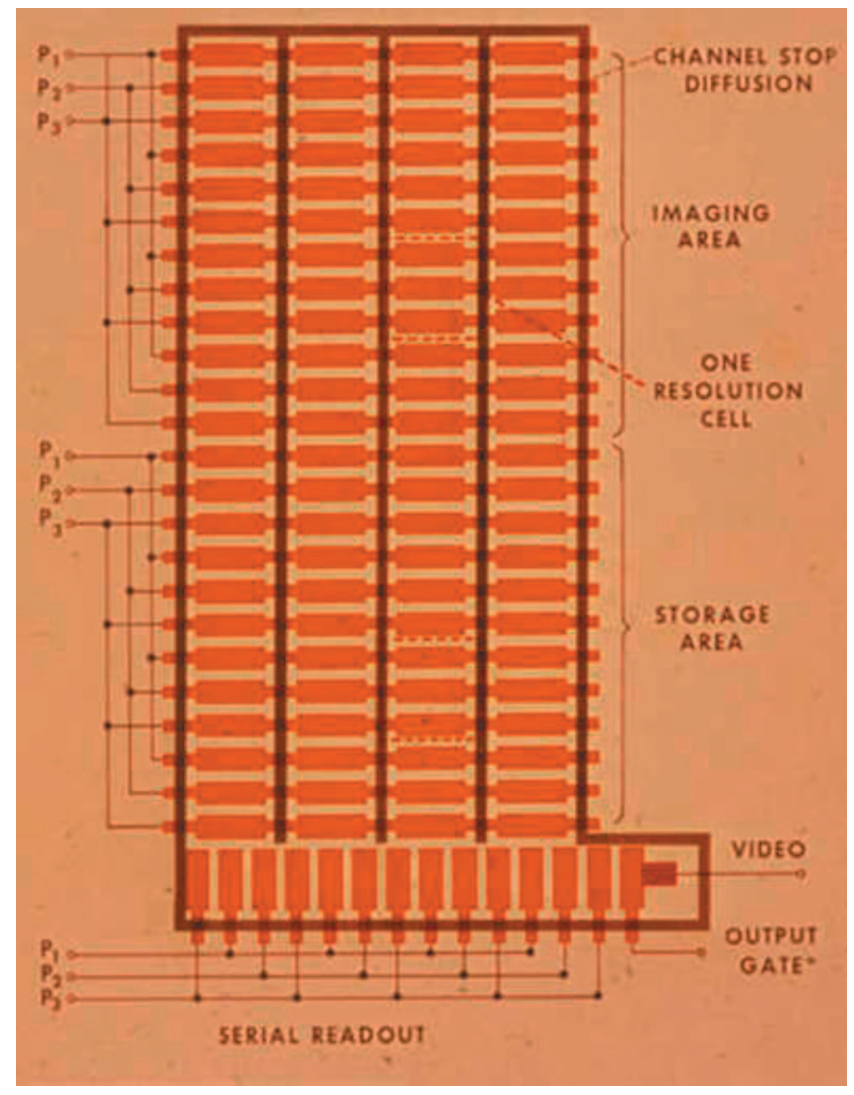

FIG. 12. (Color) An area imaging CCD geometry.

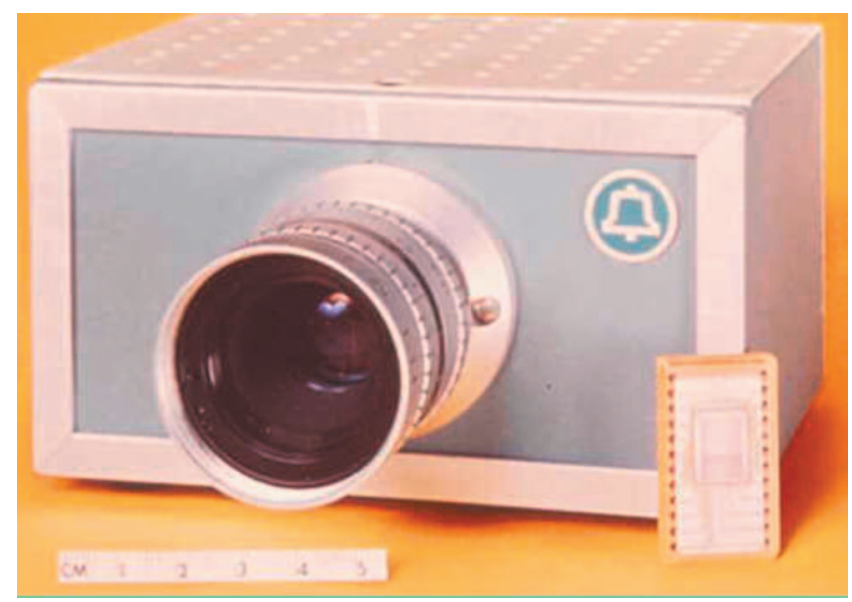

FIG. 13. (Color) The CCD Picturephone imaging chip and a self-contained camera.

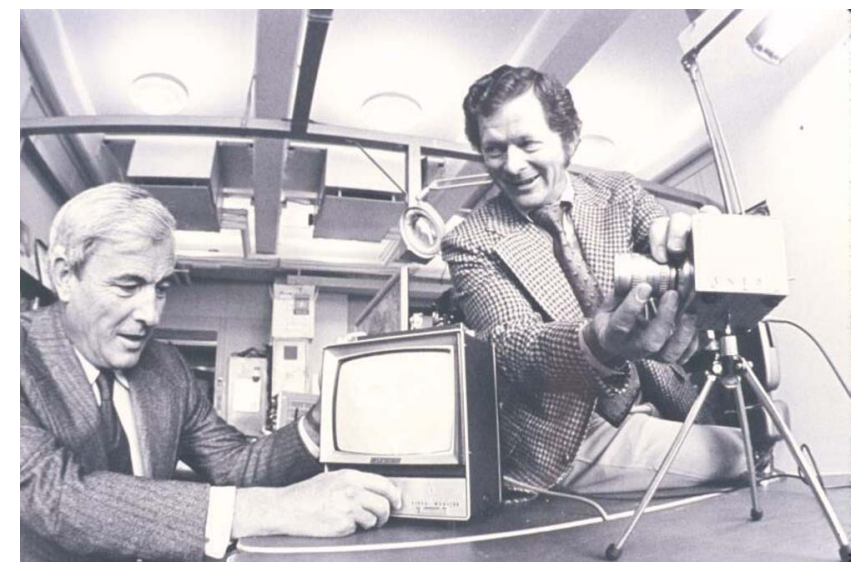

FIG. 14. (Color online) W. S. Boyle and G. E. Smith, 1970.

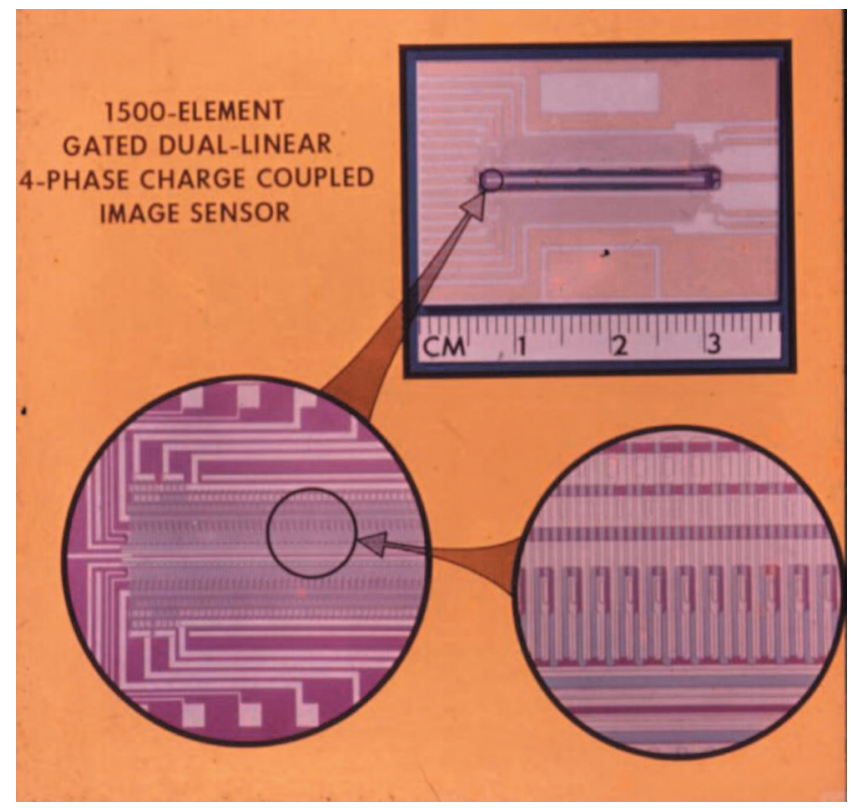

FIG. 15. (Color) An early linear imaging CCD. 


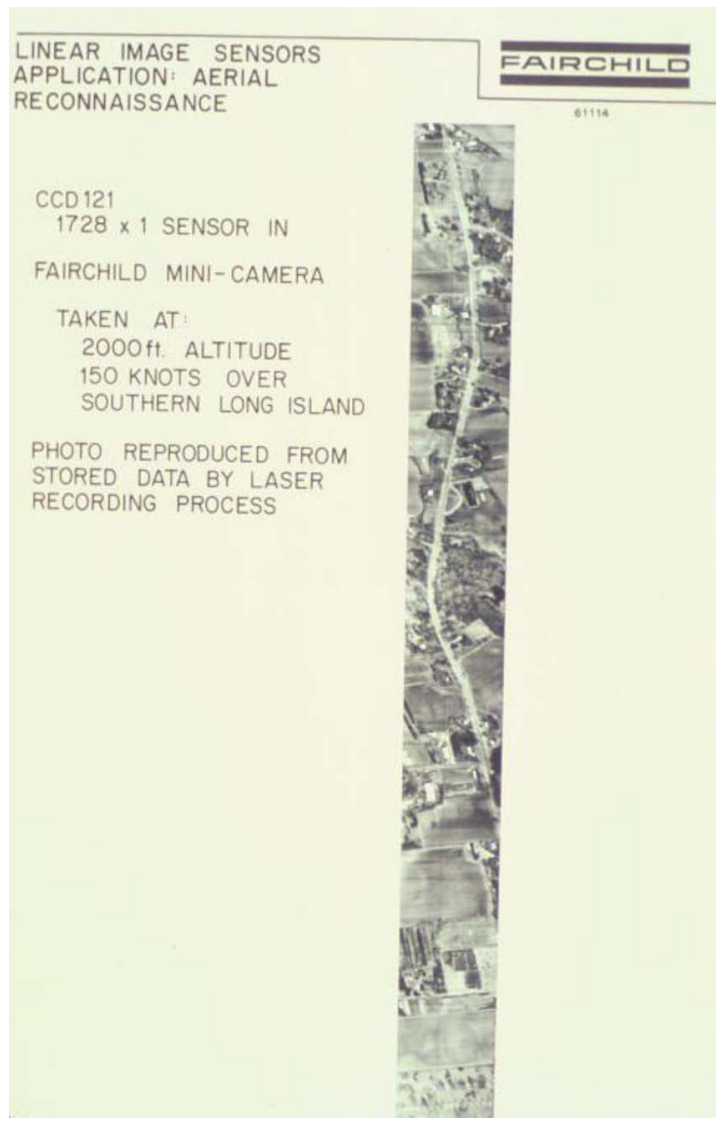

FIG. 16. (Color online) An aerial photograph taken with a Fairchild linear CCD.

inefficiency, the inability to transfer all of the charge from one element to the next. The main reason for this was the trapping of charge in traps at the silicon-silicon dioxide interface, see Fig. 10. The trapped electrons would emit at a later time causing smearing of the image. So Bill and I got together again and invented the buried channel CCD, which placed the stored charge in the interior of the semiconductor where there was relatively little trapping. The structure is shown in Fig. 11 where a lightly doped $n$ layer has been added to the original structure. Once the layer has been depleted by transferring the electrons to the output diode, the resulting potential is shown. Electrons in the channel region will now accumulate in the valley created in the interior of the silicon and kept away from the surface traps.

A period of rapid development followed both at Bell Labs and other companies. One major activity was to make an area imaging device for video applications. Many different schemes were devised. The one we chose

TABLE I. Advantages over photographic film.

$90 \%$ quantum efficiency (film is $5 \%$ )

Long integration times (at $160^{\circ} \mathrm{K}$ )

(20 electrons/h/pixel)

Large dynamic range $10^{5}$ electrons/pixel

Repeatability eliminates systematic errors

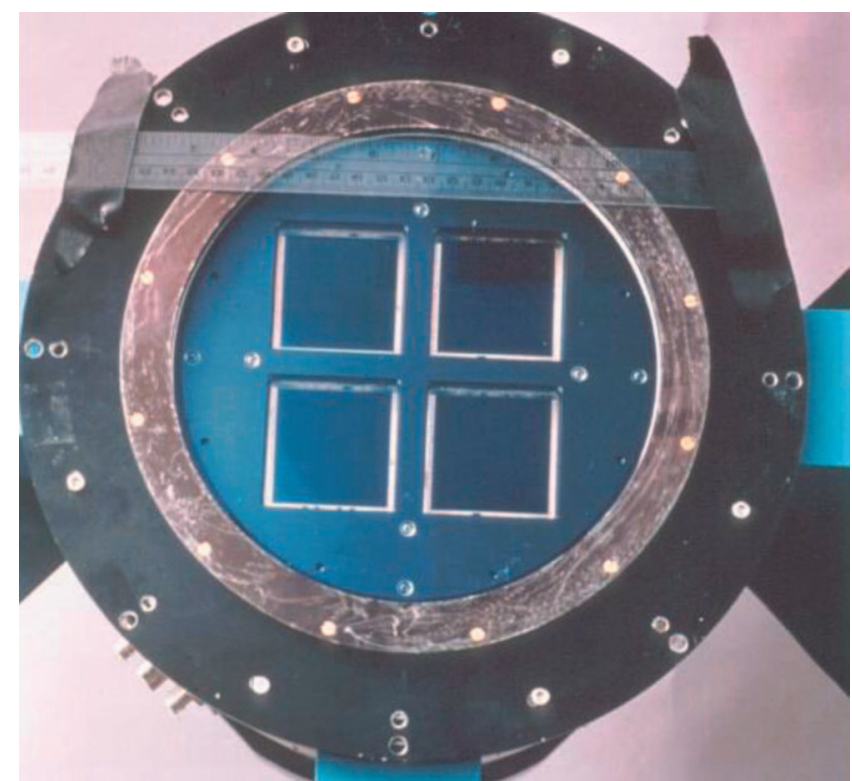

FIG. 17. (Color) An early four 1 Megabit CCD camera for an astronomical telescope.

for the Picturephone application is shown schematically in Fig. 12. Linear CCDs are formed side by side and split into an imaging and storage area. During a frame time, the image is taken in the upper region and then transferred rapidly to the lower where it is read out a line at a time by the serial readout while the next frame is being taken. The chip that we made for the Picturephone is shown in Fig. 13 along with a self-contained experimental camera. Successful testing of the device is shown in Fig. 14.

Another device that we developed in the early days was a linear scanning device shown in Fig. 15 for possible use in facsimile-type applications. Many other companies initiated strong development efforts after the initial announcement of the invention was made in early 1970 and the field rapidly became very active. An aerial photo taken with a linear scanning device made by Fairchild is shown in Fig. 16.

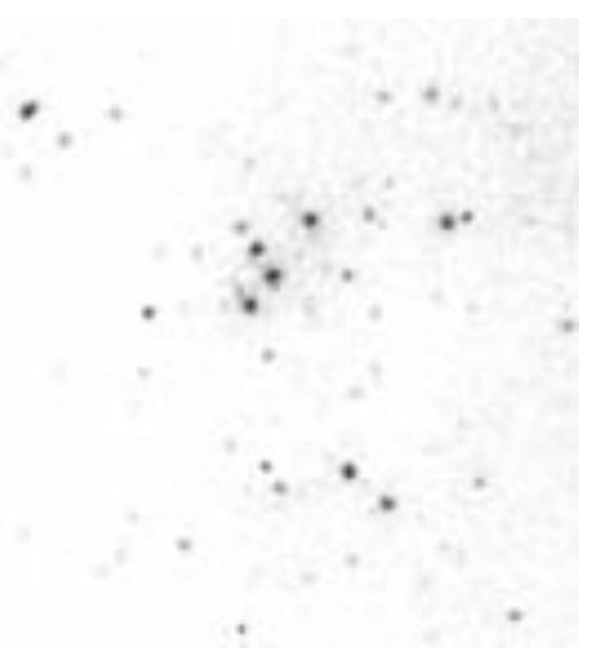

FIG. 18. A film photograph of the sky. 


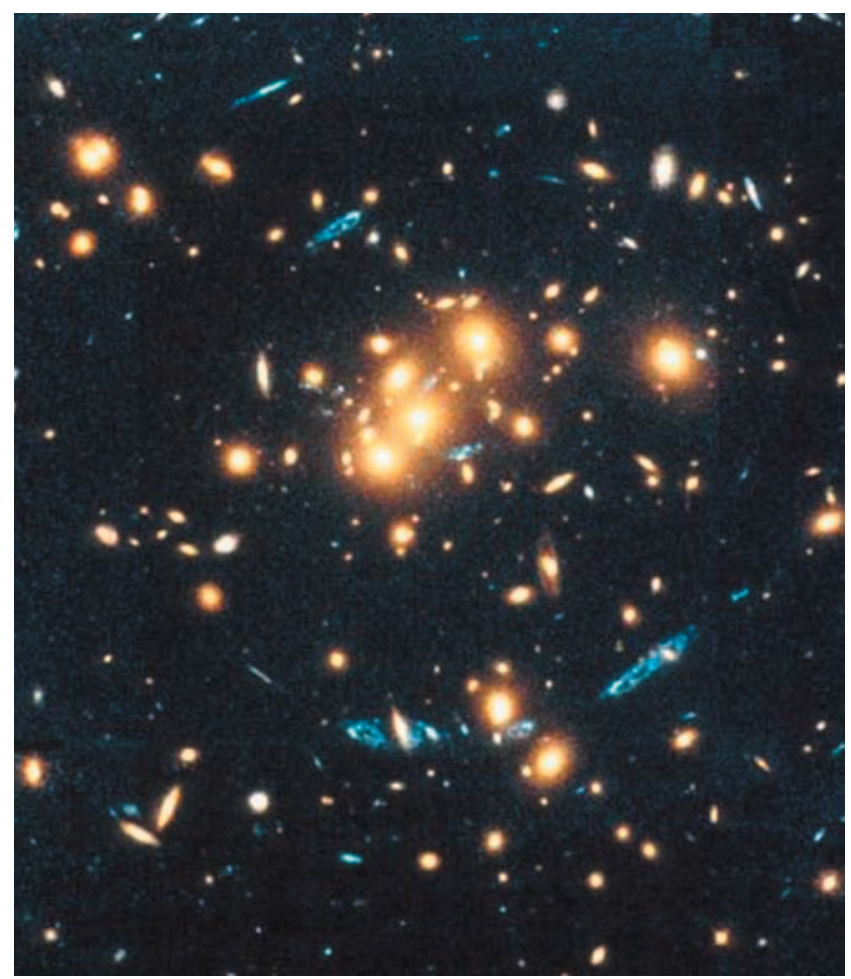

FIG. 19. (Color) A CCD image of the same portion of the sky.

Perhaps the most impressive scientific applications were in astronomy, where the impressive advantages over using photographic film listed in Table I led to many new discoveries. An early four megapixel CCD for use in an astronomical telescope is shown in Fig. 17. A comparison between a state of the art photographic im-
TABLE II. Imaging device applications of CCD.

Digital cameras

TV cameras

Scanners

Medical devices

Fax machines

Bar code readers

Satellite surveillance

Earthly surveillance

Astronomy

age, Fig. 18, compared to an image taken by a CCD, Fig. 19 of the same region, exemplifies this. Such improvements led to such things as experimental verification of dark matter in the Universe.

In summary, CCDs were born in the $\mathrm{Si}-\mathrm{SiO}_{2}$ revolution, and because of their unique properties, created their own revolution in widespread imaging device applications listed in Table II.

\section{REFERENCES}

Amelio, G. F., M. F. Tompsett, and G. E. Smith, 1970, "Experimental verification of the charge coupled device concept," Bell Syst. Tech. J. 49, 593.

Boyle, W. S., and G. E. Smith, 1970, "Charge coupled semiconductor devices," Bell Syst. Tech. J. 49, 587.

Boyle, W. S., and G. E. Smith, 1974, "Buried channel charge coupled devices," U.S. Patent No. 3,792,322 (12 February 1974). 